\title{
Tailored and tapered metallic gratings for enhanced absorption or transmission
}

\author{
Honghui Shen ${ }^{1}$, Aimi Abass ${ }^{2}$, Marc Burgelman², Bjorn Maes ${ }^{1,3}$ \\ 1 Photonics Research Group (INTEC), Ghent University-IMEC, Sint-Pietersnieuwstraat 41, B-9000 Gent, \\ Belgium. \\ ${ }^{2}$ Department of Electronics and Information Systems, Solar Cells Group, Ghent University, B-9000 Ghent, \\ Belgium. \\ ${ }^{3}$ Micro- and Nanophotonic Materials Group, University of Mons, Faculty of Science, Avenue Maistriau 19, B- \\ 7000 Mons, Belgium. (bjorn.maes@umons.ac.be)
}

\section{ABSTRACT}

Plasmonic structures, such as metallic gratings and apertures, provide unusual abilities to modify the absorption or the transmission of thin-layered devices. In order to optimize the absorption in thin-film solar cells, we introduce complex grating geometries, implementing multiple periodicities and blazing. These gratings optimize the diffraction efficiency and increase the number of accessible modes. On the other hand, in order to achieve enhanced transmission at infrared wavelengths, we propose tapered apertures. The non-resonant funnelling phenomena provide for broadband and wide-angle focusing opportunities.

Keywords: photovoltaics, plasmonics, gratings.

\section{DESCRIPTION}

Thin-film devices such as solar cells and LEDs can profit from the strong scattering effects provided by dielectric and metallic gratings [1,2].

To enhance the absorption we have optimized an amorphous silicon solar cell design in multiple ways (Fig. 1(a)). In this design we couple efficiently to the two main types of guided modes: the 'photonic' modes (mainly localized in the silicon) and the plasmonic modes (localized at the metallic back-contact). We adjust the periodicity of the front (ITO/Si) grating to couple best to the photonic modes, and we lower the period of the back ( $\mathrm{Si} / \mathrm{Ag})$ grating to diffract into plasmonic modes. In this way each grating interacts in an optimal way with the mode offering the largest field-profile overlap, leading to stronger absorption peaks (Fig. 1(b)) [3]. We rigorously simulate these effects using the frequency domain finite element software COMSOL.

In addition, for optimal plasmonic electrode designs we explore tapered, funnel-like shapes, in order to have large transmission over a broadband and wide-angle range. The tapering allows a smooth transition of the impedance, leading to non-resonant designs, which are not dominated by a few strong Fabry-Perot peaks as in more traditional structures.

(a)

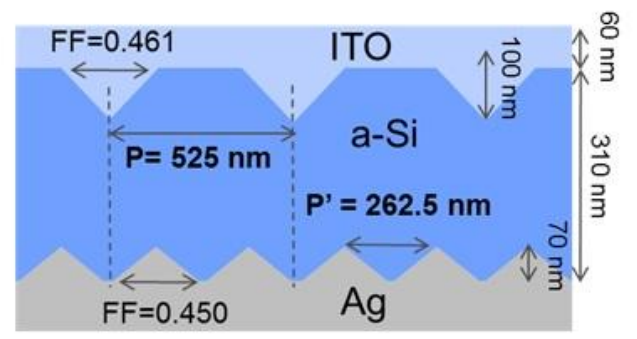

(b)

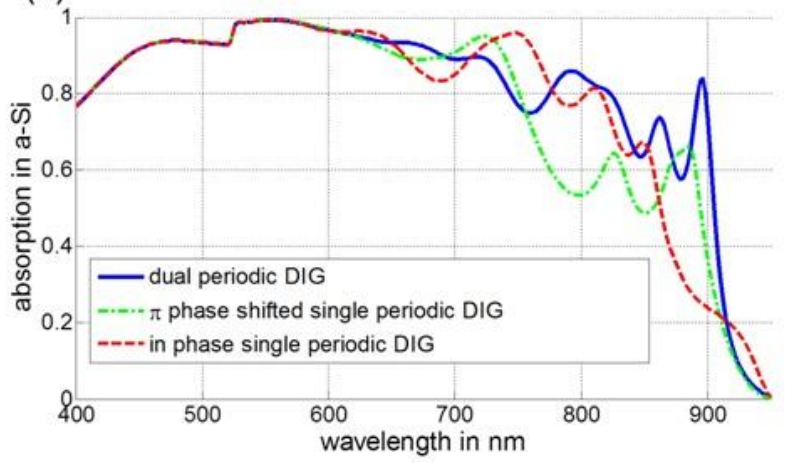

Figure 1: (a) Schematic of the dual-periodic dual-interface grating (DIG). (b) Absorption spectrum for structures with the same periodicity on both interfaces (dashed and dash-dotted), and with the period doubling (solid line). The solid line presents improved coupling to various guided modes.

ACKNOWLEDGEMENTS

We acknowledge IWT-SBO "Silasol" and IAP "photonics@be".

\section{REFERENCES}

[1] H.A. Atwater, A. Polman, Nature Materials, 9, p.205, 2010.

[2] H. Shen et al., Optics Express, 19, p.A1202, 2011.

[3] A. Abass et al., Physical Review B, 85, 115449, 2012. 